\title{
REPAIR OF BONE FOLLOWING FRACTURES *
}

\author{
JOSEPH A. BLAKE, M.D. \\ NEW YORK
}

The repair of bone following injury may seem a trite and worn out subject, yet the mass of clinical material furnished by the war has afforded opportunity for some interesting and practical observations.

During the war, there was considerable controversy, at least in France, on the old subject of production of bone by periosteum; and although I have little to say as to the activity of periosteum as a bone former, I have a strong feeling as to its value as a protector and conservator of developing bone. Portions of bone in gunshot fractures are commonly detached and driven into the muscle. Surgeons have frequently placed bone in the muscles to bridge defects. Invariably, as fas as I know, fragments, not lying in immediate contact with bone, have been gradually absorbed and have disappeared. Quite the reverse happens when bone fragments or grafts lie in close contact with bone. They form new bone almost invariably. Also, the connective tissue of repair seems to be favorable, or at least neutral, to the growth of bone, as observed in the production of osteophytic processes in muscles whose activity is violent enough to produce trauma at their insertion.

These facts suggest that while chemical conditions in the bones are favorable to growth of bone, there are substances found in the muscles which are inimical. If this is so, at least one of the functions of the periosteum may be protective in a chemical sense. The periosteum is of the greatest value mechanically in preserving the attachment of fragments of bone to the soft parts, particularly the blood vessels. In excisions of bones and joints practiced according to the method of Ollier, it carries and supports the thin shavings of bone separated by the rugine, and by transmitting the action of the muscles is one of the chief agents in restoring conformation.

Nutrition is of prime importance in the repair of bone. In fact, one may say that the rate of reproduction of bone is directly dependent on the blood supply. The fact is so evident as to make its statement superfluous, yet most of the failures of operations to unite or graft bones are due to ignorance or indifference on the part of the surgeon in regard to the sources of the blood supply. The errors of the oper-

* Read before the Pathological Society of the University of Minnesota, Sept. 28,1920 . 
ators become more evident if infection occurs. Then not only do grafts and other detached fragments become necrotic, but very frequently the ends of the main fragments, also the line of demarcation corresponding to the point at which the circulation has been interrupted. Frequently, necrosis of the entire ends occurs up to drill holes passed through the medulla. Drilling through the cortex alone, while it may lead to necrosis about the hole, does not seem to be dangerous; but

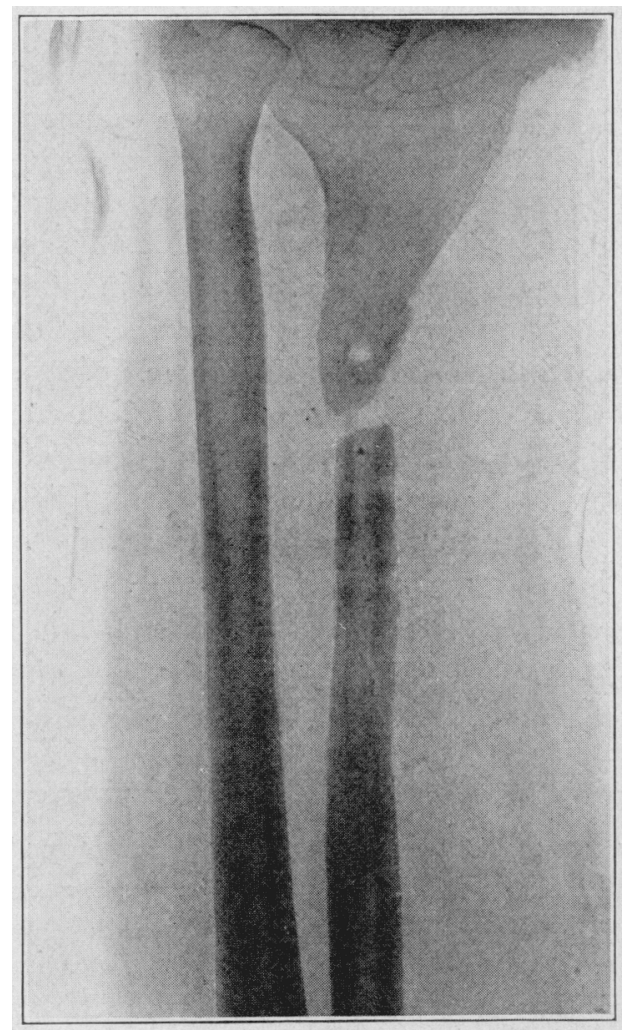

Fig. 1.-Ununited fracture of the radius six months after injury and three months after a sliding graft operation. Although there was no history of infection immediately following the operation, an abscess formed two and one-half months after the operation, discharging spontaneously under the plaster cast. Density of the bone between the drill holes denoting necrosis may be noted, also the rarefaction, particularly of the distal fragment.

interference with the medulla, the main source of blood supply, is likely in case of infection to lead to complete death (Fig. 1). In other words, inhibition of the blood supply would seem to convert the terminal portions of the fragments into bone which is no better than that transplanted from another source in the patient. 
Almost invariably, finely comminuted fractures in which we know a large part of the fragments are absolutely detached, if not infected, unite with remarkable rapidity, the resulting callus being usually excessively large. Every fragment must contribute its quota of new bone in order to produce the amount observed. I have frequently seen similar bone production from chips inserted about a graft or between the ends of the main fragments. I have also observed a new graft, inserted alongside a graft which had been in for six months and had been fractured at its middle, unite at its sides with both fragments of the original graft; the callus of union being visible at the end of three weeks by roentgenography. All of which proves that fragments of bone, even when detached, produce bone if the conditions are favorable.

When sufficient infection is present to change the chemistry of the fluids about the fragments, all detached and anemic portions of the fragments usually die. On the other hand, as we have all noticed, the presence of infection produces hyperemia in the adjacent structures which leads to rapid production (perverted repair) of bone tissue from the portions of bone not killed. In this way, excessive callus is produced. While this excessive repair is distinctly advantageous in many cases in bridging over the defects resulting from extensive necrosis, it often leads to embarrassing flasklike formations about the dead fragments which are sometimes almost impossible to cure if allowed to become pronounced (Fig. 2). The obvious treatment of such conditions is to remove the dead fragments before the cavities are formed. On the other hand, if long defects have to be bridged, it may be wise to conserve the dead fragments rather than to risk interrupting growth of new bone by their removal. Often much judgment and experience are necessary to decide on the proper moment to intervene.

When a portion of bone dies, it preserves its original density, while the remaining living bone becomes softer and less dense because of absorption of its calcareous constituents. Of the causes of this rarefaction, I shall speak later. At present, I desire to call your attention to the difference in density between the dead and living bone as the most valuable diagnostic aid in determining the presence of dead bone. In roentgenography, the dead portion, or portions, throws a much more distinct shadow. This is well shown in Figure 1. In a roentgenogram a dense portion, particularly if it has sharp and irregular contours, separated by a clear interval from the rest of the bone, is certain to be a sequestrum. Sequestra preserve their density for very long periods. I have roentgenographed and removed a number which I knew had been present for many months, and they have always been extremely hard and dense. Although bacteria invade the canaliculi as 
found by Kenneth Taylor, ${ }^{1}$ osteoclasts do not, and what sequestra lose by absorption is entirely from their surfaces. I am inclined to believe that they are not absorbed at all, for all that I have removed, whether still attached by the living bone or separated for a long time, have always had the sharp points, often long and attenuated, which are characteristic of the line of demarcation. If absorption does occur, these slender points would be lost. The cavities formed about sequestra are rather caused by absorption of the adjacent living bone, especially the involucrum. The latter thus becomes a shell, being continually added to peripherally and absorbed centrally.

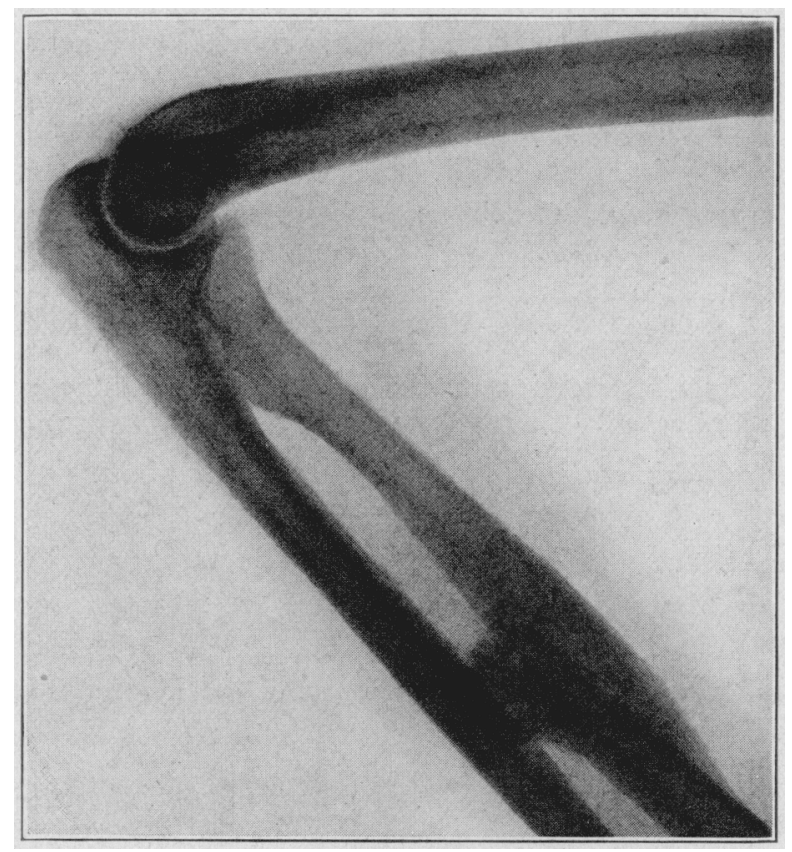

Fig. 2.--Compound fracture of the radius of eighteen months' duration. The end of the distal fragment is almost entirely necrotic. The irregular outlines of the sequestra which are somewhat denser than the involucrum, are outlined by the cavity forming about them. The excessive formation of the callus may be noted. If allowed to remain, the involucrum will be further excavated about the sequestra and a large flasklike cavity will result.

You may ask what happens to fragments of detached bone such as those projected into the muscles in a gunshot fracture if they do not die; for they disappear. My observations lead me to the conviction that they are digested alive either by their own osteoclasts or by the

1. Taylor, Kenneth: The Persistence of Bacteria in Bone Sequestra, Ann. Surg. 66:522 (Nov.) 1917. 
fluids and cells in their strange environment. If they die en masse, infection is present and they are discharged with the formation of an abscess or sinus.

I have always found rarefaction present in fractured bones, and I have found that its degree depends more on disuse than on infection. One of the chief etiologic factors in the production of rarefying osteitis as stated in our textbooks is tuberculosis. All surgeons, and I presume pathologists, are familiar with the ease with which the epiphyses of the femur and tibia can be cut in tuberculosis of the knee. Until I had operated on old gunshot fractures in the war, I had believed that tuberculosis was peculiarly productive of rarefying osteitis. In these fractures, I found the same softening of the epiphyses, fully as marked as in tuberculosis, and to a less degree, of course, of the diaphyses. The condition is readily discernible in roentgenograms, especially when compared to roentgenograms of the bones of the sound limbs, but more particularly when roentgenograms taken immediately after the injury and those of a later date are compared. It was also interesting to observe that if the patient was bedridden, rarefaction occurred in the other bones that were not used; but if he were about it occurred only in the immobilized limb. It was also much more marked when the limb was completely immobilized. Rarefaction is accompanied also by decrease in size of the bones. Infection, I do not now believe is so active a factor in producing rarefaction as I had formerly supposed. It seems to act by inhibiting function rather than by any direct toxic factor. In gunshot fractures of the humerus just above the epicondyles, the distal fragments may become almost invisible in the roentgenograms. Delayed union, or nonunion, is very common in these fractures, and rarefaction is more pronounced in such cases, proving that union is dependent on proper nutrition.

It has been my custom to defer operations for nonunion when marked rarefaction of one or both main fragments is present. Obviously an operation, such as inlay grafting, on account of the mutilation necessary in forming a groove, is almost sure to compromise what little circulation remains. It is better in such cases to arrange some sort of apparatus which will permit the patient to use his limb and regain its circulation and nutrition. When an operation is performed in such cases, a sleeve of lamellae of bone chiseled off with the periosteum is better than an inlay graft. A sliding graft should not be attempted. particularly if the bone is a small one, such as the ulna or radius. Inlay grafts from other bones and sliding grafts are indicated when the ends of the fragments are rounded over and the medullary cavity plugged by hard bone (Fig. 3). This rounding over and plugging denotes 
repair and coincidently nutrition, and is rarely seen where there is marked rarefaction. In such cases, the channel cut for the graft opens out the medullary cavities, and if the graft is taken from another bone so that active medullary tissue is removed with the graft, success can - generally be achieved.

There is another point in regard to the repair of fresh fractures which is of great importance in their treatment. I refer to the rapidity with which repair takes place. Many think that a fracture may be

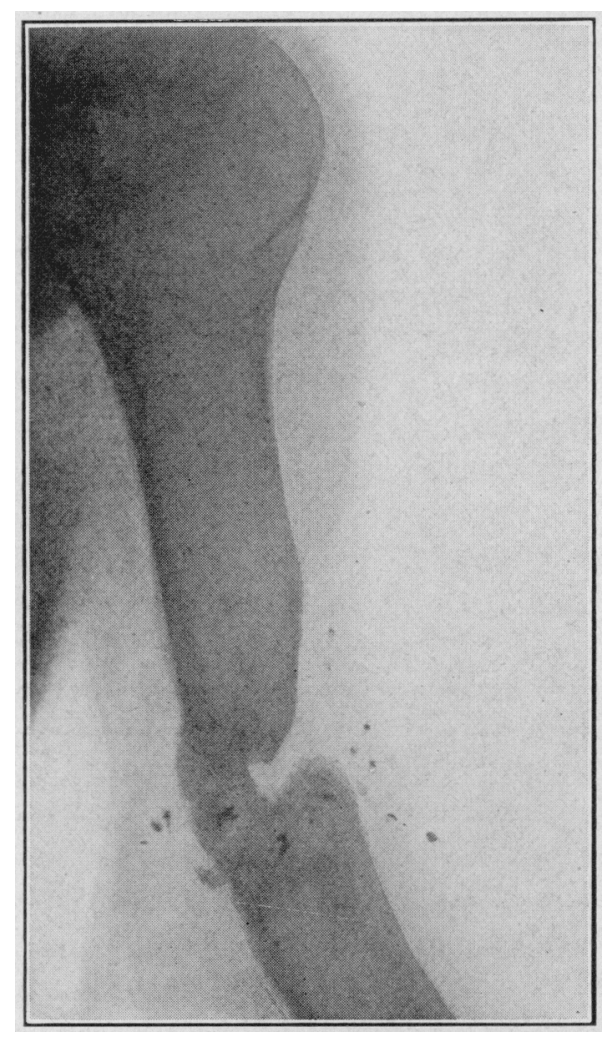

Fig. 3.-Fracture of the humerus by shell fragments, two years after injury and ten months after an attempt to unite the fragments by means of a graft, which was extruded, the wound having become infected. Complete pseudarthrosis. The rounding over of the end of the proximal fragment and the plugging of the medullary cavity of both fragments by dense bone should be noted.

reduced at any time within the first ten or twelve days. It can be, of course, but if one operates on a fracture on the fourth or fifth or sometimes even the third day, gritty new bone is found around the fragments. This increases rapidly in the next two days and if the fracture has not been reduced this new bone is not only wasted but may 
interfere seriously with reduction. Also, the soft parts repair and may prevent reduction. The custom of waiting until swelling has disappeared is a most pernicious one. It developed from the use of the plaster cast, the worst device for treating most fractures, unless used with great judgment and skill, that was ever invented. Of course, a plaster cast cannot be put on before swelling appears because it will become too tight and if put on before swelling disappears it will become too loose. A fracture should be reduced as soon as possible after it has been sustained. Often swelling will be prevented, and, of course, every bit of repair will be utilized effectively. Repair in the sense of forming new bone does not go on indefinitely. Sooner or later it ceases. To treat fractures successfully, it must be conserved and not wasted.

We have found in the treatment of fractures by suspension, together with constant active mobilization by the patient of the contiguous articulations, that the circulation is favored, that swelling rapidly disappears, that repair is rapid, and that marked rarefaction and nonunion are very rare. When splints are used, which is necessary for some fractures, they should be removed at least once, better twice a day, and the patient made to make all the motions allowable for the fracture. The appearance of the limb differs markedly from that customarily observed in treatment by splints with edematous skin with ridges here and there denoting irregular circulation. The muscles retain their form and are not atrophic. As the condition of the soft parts must be an index of that of the bony structures, it is reasonable to suppose, as seems to be proved clinically, that the circulation in the bone is far better than that obtained when the limb is kept quiet in splints. Manifestly, with better circulation, repair must be more active and there must be less tendency to necrosis of fragments devitalized because of their circulation's being cut off by traumatism or by later infection and thrombosis. Furthermore, suspension allows access to the limb for frequent dressings with the least disturbance to the fracture, which permits of better treatment and therefore quicker consolidation. Also not the least advantage of suspension in promoting circulation is the quicker sequestration of dead bone and the earlier opportunity for its removal before disagreeable callus forms.

Although nothing new has been discovered during the war in regard to the morphology of the repair bone yet much has been learned in regard to what we may expect in the formation of new bone and also in the treatment of dead bone, especially in regard to the period when it should be removed. As I hope I have made clear, it should be removed in most instances as soon as it becomes detached. In exceptional cases when it is desired that exuberant callus be formed 
to bridge over defects, it may be wise to leave in sequestra which bridge defects until the rapid callus formation which goes on about them has completed the continuity of the bone. Attention has been called to the use of dead bone for the promotion of repair by Heitz-Boyer. ${ }^{1}$ He has called attention not only to the production of new bone about grafts which have died on account of infection, but also to the stimulation caused by the introduction of dead bone from other sources. The conservation, therefore, of dead bone becomes of real practical value in many cases.

Before concluding, I should also call attention to the question as to when newly formed bone can bear the strains of use without deformation. This is of great practical importance in fractures of the femur in which the use of the limb necessitates weight-bearing, that is, a compressive force at the point of fracture. It has been found that it is not safe to allow weight-bearing on the fractured femur until the newly formed bone has established its adult condition with the formation of haversian canals. The reestablishment of the haversian system can be determined by roentgenograms.

1. Heitz-Boyer: Tr. Cong. franç. de Chir., 1919. 